\title{
Stroke-related mild cognitive impairment detection during working memory tasks using EEG signal processing
}

\begin{abstract}
The aim of the present study was to reveal markers from the electroencephalography (EEG) using approximation entropy (ApEn) and permutation entropy (PerEn). EEGs' of 15 strokerelated patients with mild cognitive impairment (MCI) and 15 control healthy subjects during a working memory (WM) task have EEG artifacts were removed using a wavelet (WT) based method. A t-test ( $\mathrm{p}<; 0.05$ ) was used to test the hypothesis that the irregularity (ApEn and PerEn) in MCIs was reduced in comparison with control subjects. ApEn and PerEn showed reduced irregularity in the EEGs of MCI patients. Therefore, ApEn and PerEn could be used as markers associated with MCI detection and identification and the EEG could be a valuable tool for inspecting the background activity in the identification of patients with MCI.
\end{abstract}

Keyword: Electroencephalography; ICA-WT; Approximation entropy; Permutation entropy; Dementia; Mild cognitive impairment; Working memory 